\title{
Antiserum Preparation of Recombinant Sweet Potato Latent Virus-Lotus (SPLV-Lotus) Coat Protein and Application for Virus-Infected Lotus Plant Detection
}

\author{
Zhen He $\mathbb{D}^{1,2}$, Tingting Dong ${ }^{1}$, Wen Chen ${ }^{1}$, Tielin Wang ${ }^{3}$, Haifeng Gan ${ }^{1}$, and LiangJun Li ${ }^{1,2 *}$ \\ ${ }^{I}$ School of Horticulture and Plant Protection, Yangzhou University, Yangzhou 225009, China \\ ${ }^{2}$ Joint International Research Laboratory of Agriculture and Agri-Product Safety of Ministry of Education of China, \\ Yangzhou University, Yangzhou 225009, China \\ ${ }^{3}$ National Resource Center for Chinese Materia Medica, China Academy of Chinese Medical Sciences, State Key Labo- \\ ratory Breeding Base of Dao-di Herbs, Beijing 10070, China
}

(Received on March 5, 2020; Revised on November 16, 2020; Accepted on November 17, 2020)

Lotus is one of the most important aquatic vegetables in China. Previously, we detected sweet potato latent virus from lotus (SPLV-lotus) and found that it has highly significant sequence diversity with SPLV-sweet potato isolates (SPLV-sp). Here, we developed serological methods for the detection of SPLV-lotus in Chinese lotus cultivation areas. Based on the high sensitivity of SPLV-lotus coat protein antiserum, rapid, sensitive and large-scale diagnosis methods of enzyme-linked immunosorbent assay (ELISA) and dot blot in lotus planting area were developed. The established ELISA and dot blot diagnostic methods can be used to detect SPLVlotus from samples successfully. And our results also showed that the SPLV-lotus and sweet potato isolates appeared clearly distinction in serology. Our study provides a high-throughput, sensitive, and rapid diagnostic method based on serology that can detect SPLV on lotus, which is suggested to be included in viral disease management approach due to its good detection level.

\footnotetext{
*Corresponding author.

Phone) +86-514-87979394, FAX) +86-514-87347537

E-mail) ljli@yzu.edu.cn

ORCID

Zhen $\mathrm{He}$

https://orcid.org/0000-0003-2460-1583

(c) This is an Open Access article distributed under the terms of the Creative Commons Attribution Non-Commercial License (http:// creativecommons.org/licenses/by-nc/4.0) which permits unrestricted noncommercial use, distribution, and reproduction in any medium, provided the original work is properly cited.
}

Articles can be freely viewed online at www.ppjonline.org.
Keywords : lotus, serological detection, sweet potato latent virus

Handling Editor : Ho-Jong Ju

Lotus (Nelumbo nucifera Gaertn.) is a member of the family Nymphaeaceae. Lotus is a perennial aquatic plant mainly distributed in China. Lotus is rich in carbohydrates, protein, vitamins and other substances, and it has high edible and medicinal value (Chiang and Luo, 2007; Masuda et al., 2007; Yu et al., 2013). At present, there are few reports on viral diseases on lotus. A previous study has found that viral diseases in lotus are caused by cucumber mosaic virus (CMV) and dasheen mosaic virus (DsMV) (Yu et al., 2015). More recently, based on high-throughput sequencing, we detected and identified apple stem grooving virus (ASGV) (He et al., 2019) and sweet potato latent virus (SPLV) (Wang et al., 2019) in lotus. SPLV belongs to the genus Potyvirus of the family Potyviridae (Wang et al., 2013). During viral disease suvery of lotus in 2017, the SPLV detection rate reached $62.7 \%$, which indicated a wide distribution of sweet potato latent virus from lotus (SPLV-lotus) in Jiangsu Province, China (Wang et al., 2019). And our previous report showed that the polyprotein encoding region (the large ORF) nucleotide similarity between SPLV-lotus and SPLV sweat potato isolates (SPLV$\mathrm{sp}$ ) are around $76 \%$ (Wang et al., 2019), which is consistent with the classification criteria of family Potyviridae (Wang et al., 2019; Wylie et al., 2017).

There are many types of plant viruses, which have a great impact on the yield and quality of crops. The premise of 
solving virus hazards is to establish a convenient, efficient and fast virus detection system. Serological methods, also known as immunological methods, use the specific binding of an antigen to an antibody to detect a plant virus (Clark and Adams, 1977; Xie et al., 2013). The advantages of this method are mainly high sensitivity, high accuracy, simple operation, and intuitive observation (Boonham et al., 2014). Compared with other detection methods, enzymelinked immunosorbent assay (ELISA) has low cost, good reproducibility, and can process multiple samples at one time, so it is one of the preferred initial detection methods for large sample sizes (Engvall and Perlmann, 1971). The most commonly used methods are ELISA (Boonham et al., 2014; Clark and Adams, 1977), dot immunoassay (Tsai et al., 1989), electrochemical ELISA (Bhimji et al., 2013). In this study, we have detected SPLV-lotus by reverse transcription polymerase chain reaction (RT-PCR) (Wang et al., 2019). SPLV-lotus coat protein (CP) was amplified by RT-PCR, cloned and expressed in Escherichia coli. Recombinant $\mathrm{CP}$ was purified and used to generate polyclonal antisera in New England white rabbits. We have established standard, sensitive and high-throughput assays such as western blot, dot blot and ELISA. The study provides reliable, high-throughput assays for SPLV-lotus antiserum in the field of Chinese lotus planting areas.

Source of plant material. Lotus samples showing late germination, early dormancy, declined growth, thin body, deep color, spots, and stripe symptoms were collected from a plantation in Yangzhou city, China (119.4E, 32.4N). Sweat potato samples were collected from Xuzhou city, China (117.3E, 34.2N). Both lotus and sweat potato samples infected by SPLV were confirmed by RT-PCR. The detection primers for lotus are CP-F (5'-GCTGCTGCTGACGATGAACTAGA-3') and CP-R (5'-CTAATGAATGCCACGCATTCCAA-3') to detect SPLV-lotus CP coding region (reference sequence: $\mathrm{MH705333).} \mathrm{The} \mathrm{detection}$ primers for sweat potato are SPLV-SP-CP-F (5'-CAGATAAGGGTAAGGAAGT-3') and SPLV-SP-CP-R (5'-GCATTCCAAGTAGTGTGT-3') to detect SPLV-sp $\mathrm{CP}$ coding region (reference sequence: $\mathrm{NC}$ 020896).

Expression and purification of CP. The coding sequence for the CP gene was amplified from the cDNA of SPLV-lotus infected lotus plants using CP- $\mathrm{F}_{\text {Ncol }}\left(5^{\prime}-\mathrm{CATGCCATG-}\right.$ GCTGCTGCTGACGATGAACTAGA-3') and CP-R ${ }_{\text {Sac }}$ (5'-CGAGCTCGTCTAATGAATGCCACGCATTCCAA- $\left.3^{\prime}\right)$. The resulting PCR products were inserted into the same sites of pET28(a), and the construct was transferred into E. coli strain Rosetta (DE3). The Rosetta cells carrying pET28(a)-SPLV-lotus CP were cultured in LuriaBertani (LB) to harvest a large amount of protein medium containing $50 \mathrm{~g} / \mathrm{ml}$ kanamycin at $37^{\circ} \mathrm{C}$, and $0.1 \mathrm{mM}$ IPTG was added into the culture. The bacterial liquid was centrifuged, and the precipitate was suspended with protein purification buffer $(20 \mathrm{mM}$ Tris- $\mathrm{HCl}, \mathrm{pH} 8.8,500 \mathrm{mM} \mathrm{NaCl}$, 10\% glycerol, $1 \mathrm{mM}$ PMSF). The ultrasonic breaker (Model 500 homogenizer, Fisher Scientific, Houston, TX, USA) was used to disrupt the bacterial cells, and the remaining liquid was centrifuged for harvesting the clarified supernatant. Obtained supernatants were incubated with Ni-NTA Sefinose resin (Sangon Biotech, Carlsbad, CA) in a glass column, and the column was eluted with stepwise increase in the concentration of imidazole. The eluted recombinant SPLV-lotus coat proteins were condensed with Ultra-15 filter unit (Millipore, Billerica, MA, USA) and used for next immune New England white rabbits.

Sodium dodecyl sulfate polyacrylamide gel electrophoresis (SDS-PAGE) and western blotting analysis. Purified protein was mixted with equal volume of $2 \times$ SDS loading buffer, then heated in boiled water for $10 \mathrm{~min}$. The treated sample was loaded onto a $12.5 \%$ SDS-PAGE for electrophoresis. Protein bands were visualised by Coomassie Blue R250 staining. For western blot, the purified samples were separated on $12.5 \%$ SDS-PAGE and transferred to the polyvinylidene fluoride (PVDF) membrane (GE Healthcare, Pittsburgh, PA, USA). The prepared CP antiserum above was taken as the first antibody (diluted $1: 5,000)$ to detect the CP in sample.

Dot blot, ELISA quantification, and data analysis. For dot blot, liquid nitrogen-freezed fresh leaves were ground to a powder, and the powder was dissolved in 2 equal volumes $(\mathrm{m} / \mathrm{v})$ of $0.02 \mathrm{mM}$ PBS buffer $(0.02 \mathrm{M} \mathrm{NaCl}, 0.02$ $\left.\mathrm{M} \mathrm{KH}_{2} \mathrm{PO}_{4}, 0.02 \mathrm{M} \mathrm{Na}_{2} \mathrm{HPO}_{4}, 0.02 \mathrm{M} \mathrm{KCl}\right)$. The prepared PVDF membrane was wetted with $0.02 \mathrm{M}$ PBST buffer and air dried before loading. Each sample supernatant (5 $\mu l)$ was spotted in the center of the small square and air dried. The diluted SPLV-lotus CP was used as a positive control, and a healthy lotus sample was used as a negative control. After the completion of a chromogenic reaction, the film was photographed, and the image was used for further quantitative analysis. For the ELISA assay, the ELISA samples were prepared similarly to the dot blot. Results were recorded at $\mathrm{OD}_{450}$ using an ELISA reader (Biotek EL X 800, Winooski, VT, USA).

Image $\mathrm{J}$ software was used to quantify the intensity of the reaction color in the PVDF membrane. The independent dot blot films were measured 3 times, respectively, and the 
final date is the average of the measurement results. The software performed a detailed operation according to the instructions, and the measured value indicated the intensity of the point color that appears. The date obtained from the ELISA reader was exported to Excel software, and the average reading for each sample was obtained based on the position on the plate. The criteria for positive ELISA results could be described as follows: the positive control reading minus the blank reading are above two times of the data that negative control reading minus the blank reading.

Cloning, evaluation, and purification of SPLV-lotus CP gene. We amplified the SPLV-lotus CP gene with primers CP-FNcoI/CP-RSacI by RT-PCR and constructed by double digestion to the pET28(a) vector. SPLV-lotus encodes a polyprotein that forms a mature $\mathrm{CP}$ upon hydrolysis. The mature SPLV-lotus CP contains 294 amino acids with an isoelectric point of 6.8. The constructed pET28(a)-CP was

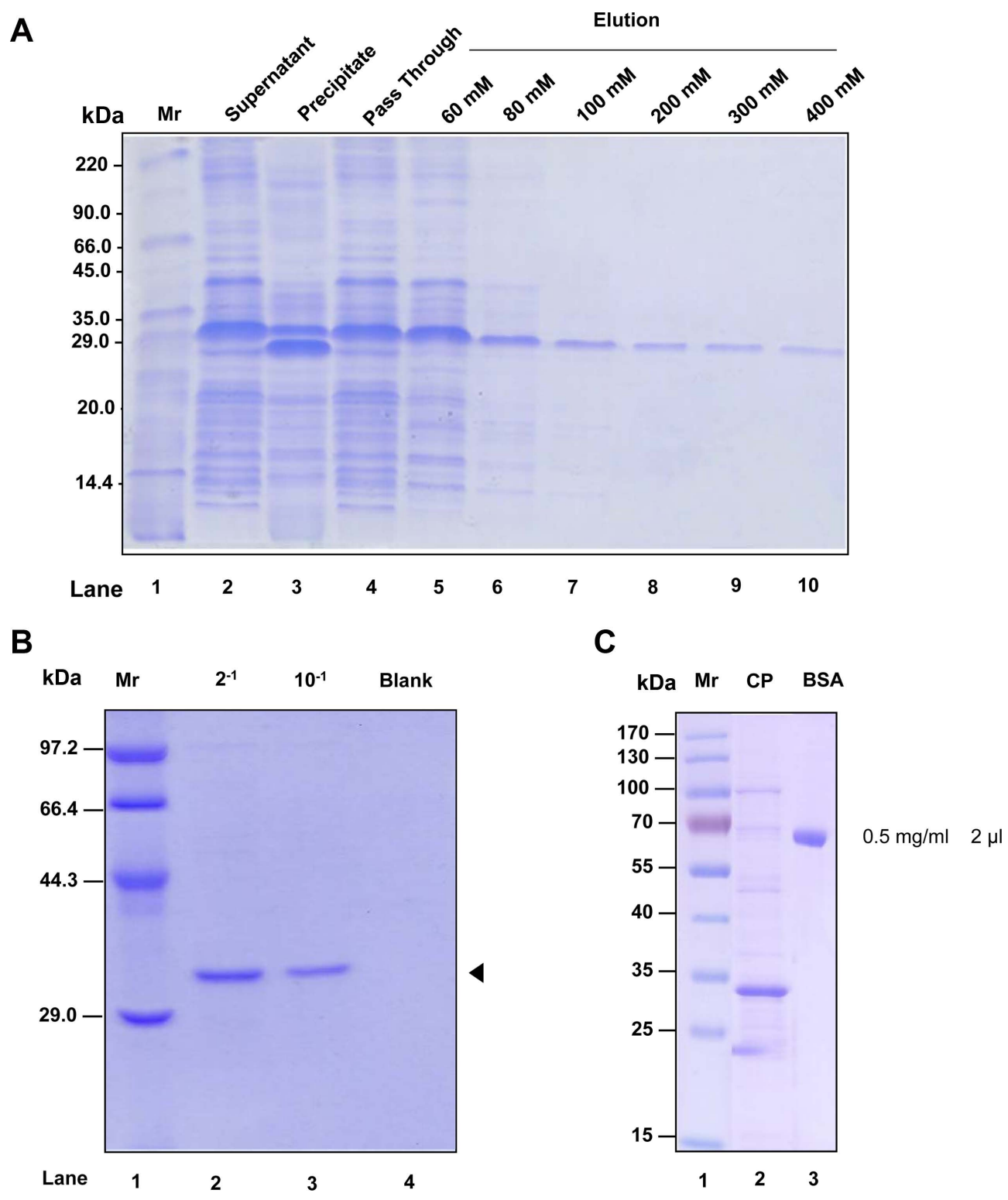

Fig. 1. Prokaryotic expression and quantification of recombinant coat protein (CP). (A) Analysis of the purification efficiency of sweet potato latent virus from lotus (SPLV-lotus) CP recombinant protein through imidazole elution. (B) Quantification analysis of condensed $\mathrm{CP}$ protein by Coomassie bright blue staining. Lane 2, the condensed SPLV-lotus CP protein was diluted twice, and the loading volume of SPLV-lotus CP protein was $10 \mu \mathrm{l}$; lane 3, 10 times dilution of the condensed SPLV-lotus CP protein, and $10 \mu \mathrm{l}$ of SPLV-lotus CP protein was loaded. (C) Quantification of the condensed SPLV-lotus CP protein by $0.5 \mathrm{mg} / \mathrm{ml}$ bovine serum albumin (BSA). Lane 2, 2 $\mu \mathrm{l}$ condensed SPLV-lotus CP protein; lane $3,2 \mu 10.5 \mathrm{mg} / \mathrm{ml} \mathrm{BSA}$. 
transformed into the E. coli Rosetta (DE3) strain. Positive clones were grown in liquid LB medium for further protein expression and purification. We induced protein expression using $1 \mathrm{mM}$ IPTG. After an elution test with increasing imidazole concentration (Fig. 1), the highest elution efficiency of the target recombinant protein was achieved in $200 \mathrm{mM}$ imidazole (Fig. 1A, lane 8), and $200 \mathrm{mM}$ was selected in the next step. An Ultra-15 filter unit (Millipore) with a molecular weight greater than $30 \mathrm{kDa}$ was used to concentrate and further purify the target. Analysis of the concentrated protein by SDS-PAGE and bright blue staining (Fig. 1B) showed that our purification method was successful. We quantified the purified recombinant SPLV-lotus CP with 0.5 $\mathrm{mg} / \mathrm{ml}$ bovine serum albumin as a control, and our protein concentration was similar to BSA (Fig. 1C, lanes 2 to 3). These results indicate that our protein purification method is correct and the purified SPLV-lotus CP is relatively clean. The quantity and total amount of SPLV-lotus CP met the requirements for polyclonal antiserum preparation conditions and used for the immunization of New England white rabbits.

Evaluation of prepared antibody titer for SPLV-lotus. The purified recombinant SPLV-lotus CP was used as an antigen, and the prepared antiserum was used as an antibody for ELISA. The concentration of the CP was diluted to $1 \mu \mathrm{g} / \mathrm{ml}, 96$-well polystyrene plates were covered overnight at $4{ }^{\circ} \mathrm{C}$, the wells were blocked with $1 \%$ BSA solution, and then captured with increasing amounts of diluted SPLV-lotus CP antiserum. 0.01\% horseradish peroxidaseconjugated IgG dissolved in 0.02 PBST buffer was used to capture the first antibody. After 10 minutes color reaction at $37^{\circ} \mathrm{C}$, a reading was taken at $\mathrm{OD}_{450}$. When the dilution ratio of $\mathrm{CP}$ antiserum was 1:64000, the $\mathrm{OD}_{450}$ reading remained

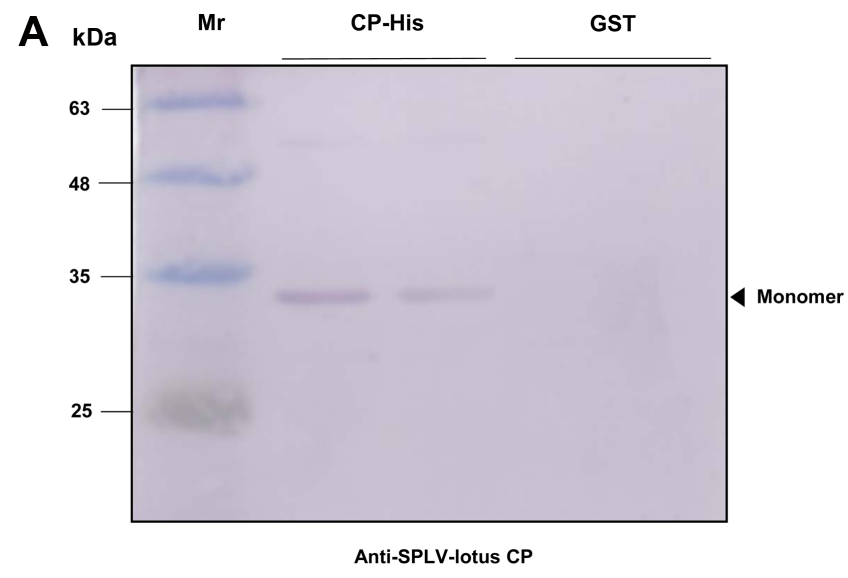

\section{B}

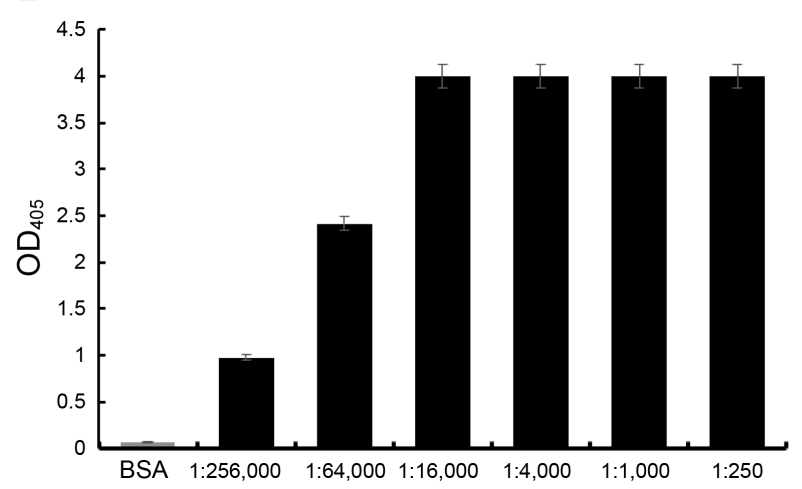

Fig. 2. Evaluation of prepared antibody titer for sweet potato latent virus from lotus (SPLV-lotus) coat protein (CP). (A) Enzyme-linked immunosorbent assay was used for the detection of the prepared polyclonal antibody titer of SPLV-lotus CP recombinant protein. (B) Western blot analysis for the evaluation of prepared polyclonal antiserum of SPLV-lotus CP protein. GST indicates purified GST protein, which were considered as controls. CP-His indicates purified SPLV-lotus CP recombinant protein.

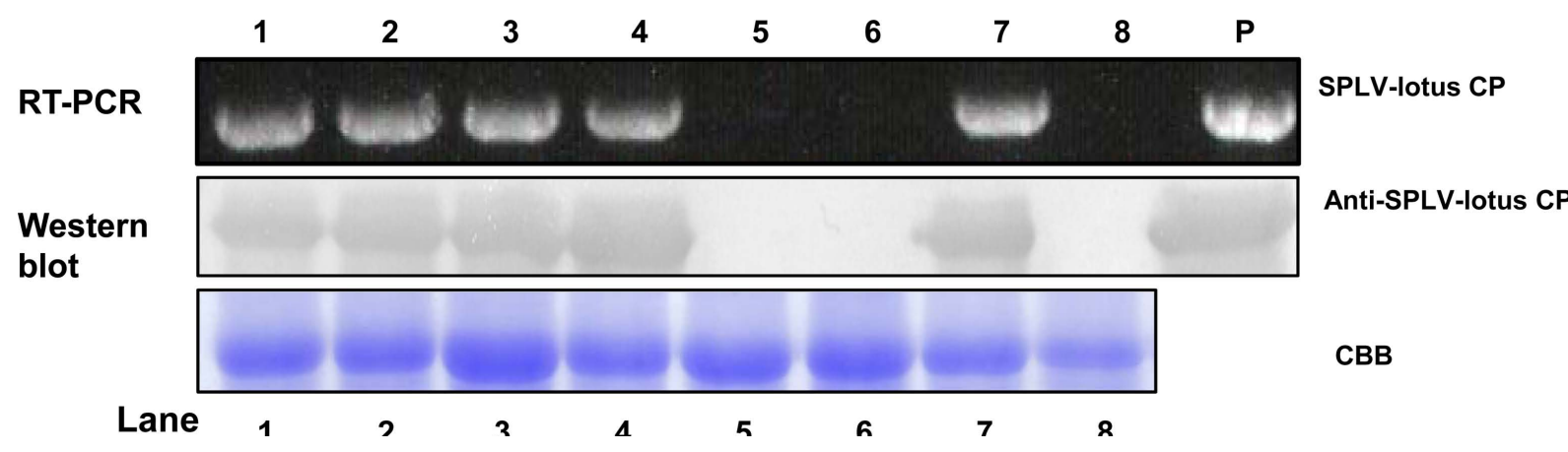

Fig. 3. Western blot analysis for the detection of lotus samples from wild field. Total proteins were stained by Coomassie bright blue and considered as loading control. Number 1-8 are the lotus diseased and healthy samples collected from the field. CBB, Coomassie bright blue; CP, coat protein; P, positive sample; RT-PCR, reverse transcription polymerase chain reaction; SPLV-lotus, sweet potato latent virus from lotus. 
higher than 0.6 (Fig. 2A). The ELISA evaluation of CP antiserum indicated that the prepared antiserum was qualified and could be used for the development of serological-based molecular detection methods. To verify the specificity of the prepared polyclonal $\mathrm{CP}$ antiserum, purified $\mathrm{CP}$ was also used as an antigen for western blot analysis. Purified GST protein was used as a negative control, and purified CP-His was used as a detection target. The specific bands $(32 \mathrm{kDa})$

A

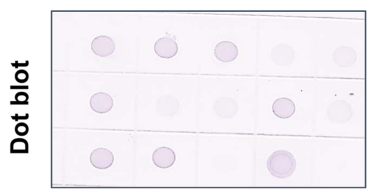

\begin{tabular}{|c|c|c|c|c|}
\hline 1 & 2 & 3 & 4 & 5 \\
\hline 6 & 7 & 8 & 9 & 10 \\
\hline 11 & 12 & N & P & $M$ \\
\hline
\end{tabular}

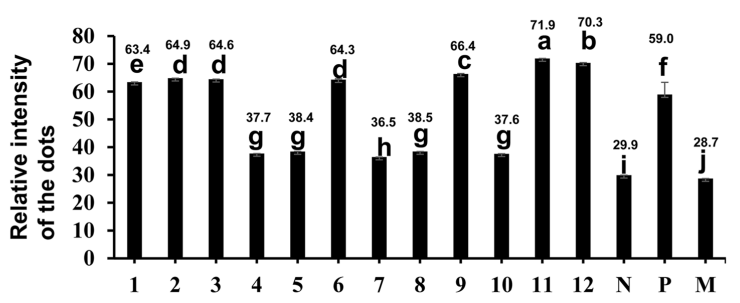

B
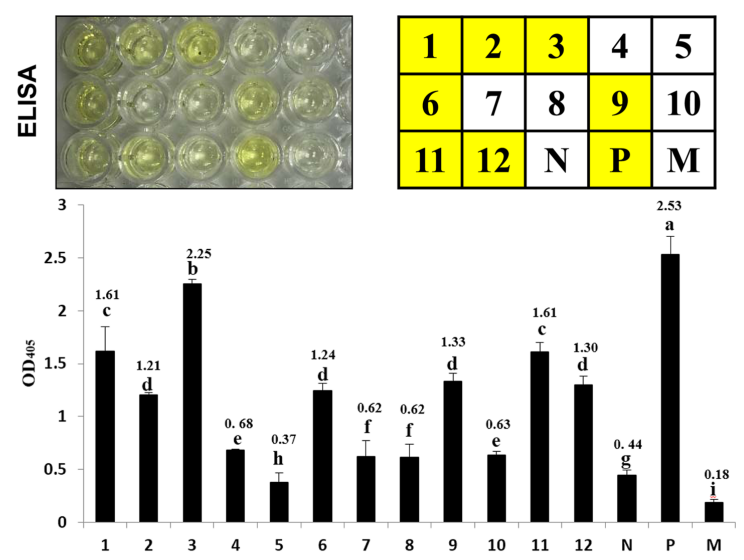

C

$\begin{array}{llllllllllllllll}M r & 1 & 2 & 3 & 4 & 5 & 6 & 7 & 8 & 9 & 10 & 11 & 12 & \mathrm{~N} & \mathrm{P} & \mathrm{M}\end{array}$

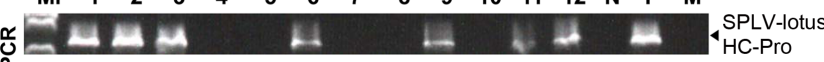

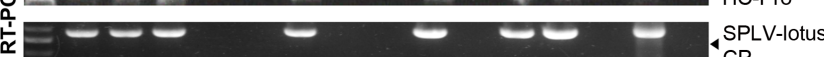

Fig. 4. Dot blot and enzyme-linked immunosorbent assay (ELISA) detection of the lotus samples from wild field. (A) Dot blot for the detection of the 12 lotus samples from independent plants. The intensity of the stained dot was quantified. Error bar standard deviation (SD) represents the means of independent lotus samples from the same row (Student's T-test, $P<0.05$ ). P, positive sample; $\mathrm{N}$, negative sample; $\mathrm{M}$, blank control. (B) ELISA was developed for the specific and high-throughput diagnosis of the 12 lotus samples from wild field. The samples, quantified data analysis, and statistical methods were similar to the dot blot assay. (C) Reverse transcription polymerase chain reaction (RTPCR) results of 12 lotus samples. CP, coat protein; SPLV-lotus, sweet potato latent virus from lotus. were clearly visible from the film (Fig. 2). Western blot results further indicated that the prepared polyclonal $\mathrm{CP}$ antiserum was qualified and specific for SPLV-lotus CP detection.

Serological detection of SPLV-lotus by Western blot, dot blot, and ELISA. To demonstrate the utility of CP antiserum, we first collected 8 fresh lotus samples from Yangzhou city Jiangsu province. Eight lotus samples including health control were ground to powder for western blot detection and RT-PCR detection. Western blot revealed a strip-specific band, and the RT-PCR results were consistent with western blot analysis (Fig. 3). The total volumetric loading of each lane was shown by total protein staining. To achieve high-throughput and rapid detection of wild

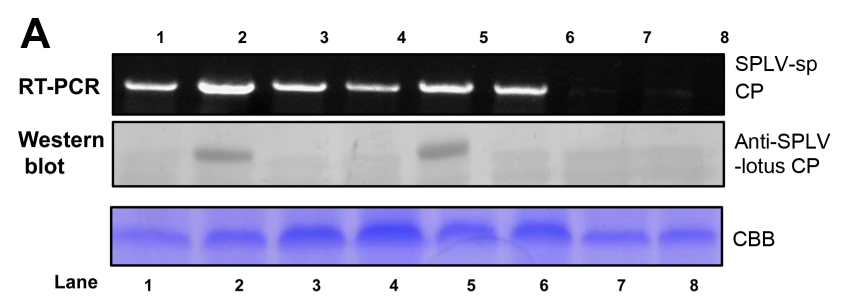

B

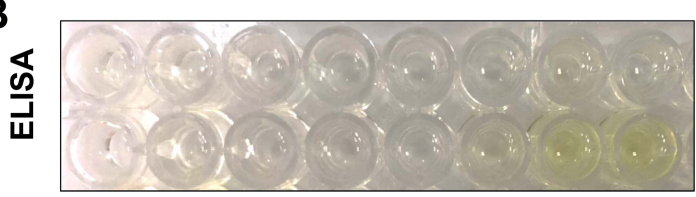

\begin{tabular}{|c|c|c|c|c|c|c|c|}
\hline 1 & 2 & 3 & 4 & 5 & 6 & 7 & 8 \\
\hline 9 & 10 & 11 & 12 & N & N & P & P \\
\hline
\end{tabular}
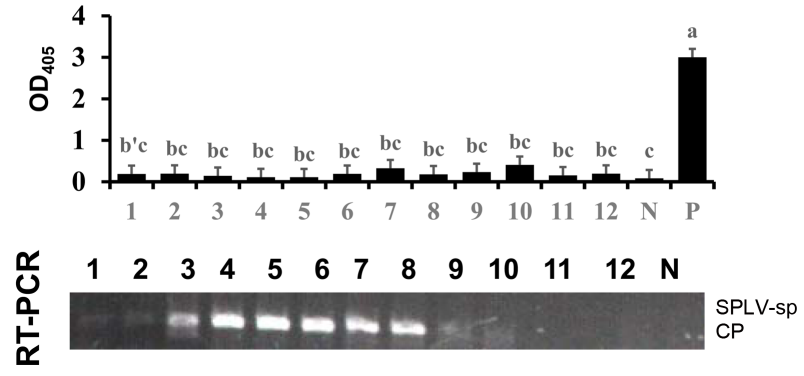

Fig. 5. Western blot (A) and enzyme-linked immunosorbent assay (ELISA) (B) detection of the sweet potato samples from field using sweet potato latent virus from lotus (SPLV-lotus) coat protein (CP) antiserum. Total proteins were stained by Coomassie bright blue (CBB) and taken as loading control. Error bar standard deviation (SD) represents the means of independent lotus samples from the same row (Student's T-test, $P<0.05$ ). N, negative sample; P, positive sample; RT-PCR, reverse transcription polymerase chain reaction; SPLV-sp, sweet potato latent virussweet potato isolates. 
lotus by the prepared CP antiserum, we also developed dot blot and ELISA methods. We re-collected 12 fresh lotus samples from Yangzhou city. We chose uninfected lotus as a negative control and diluted the protein sample as a positive control. On the dot blot film, the intensity of the spots representing the tissue samples was different (Fig. 4A). These points were quantified by Image $\mathrm{J}$ software, and the
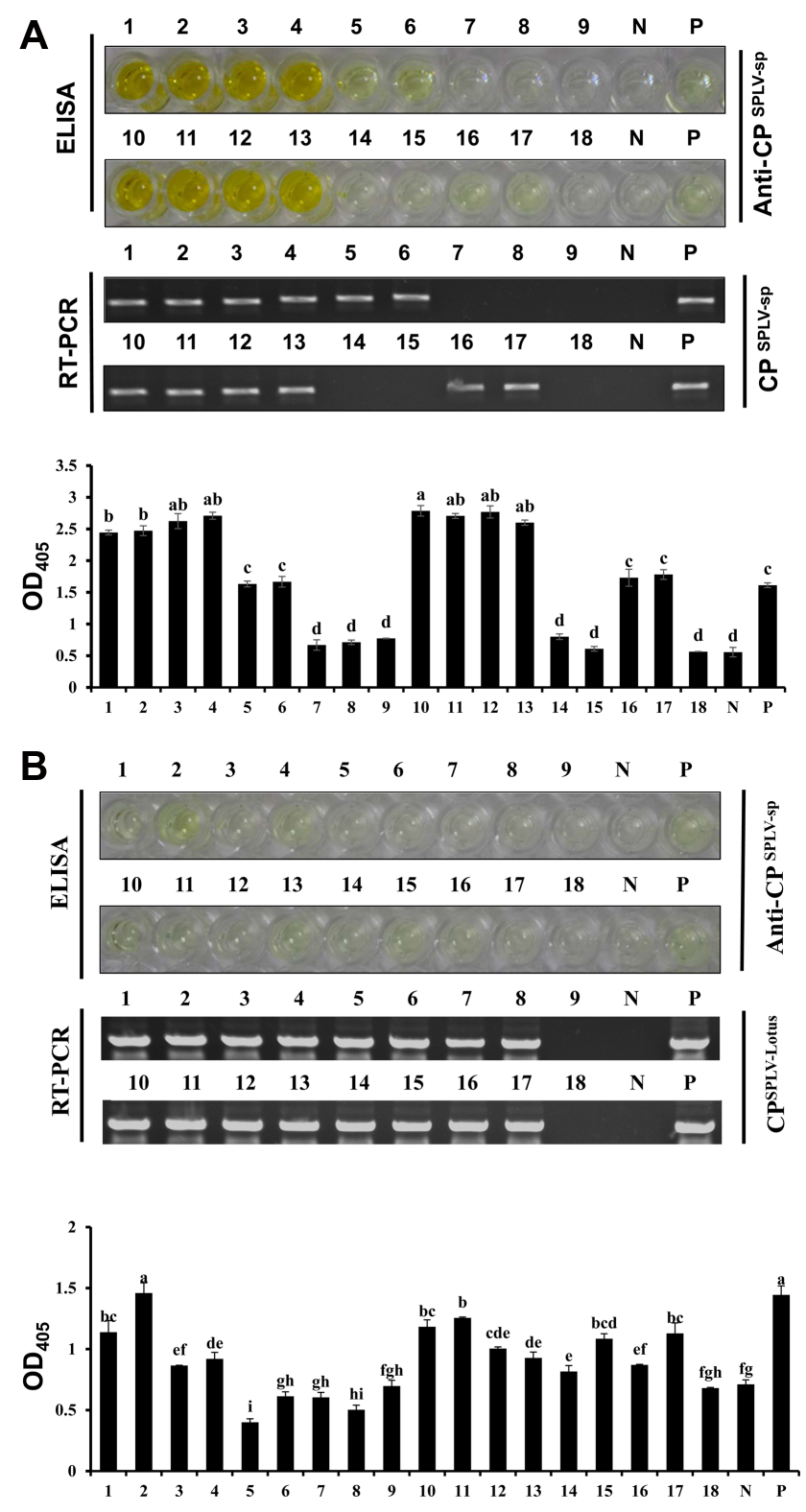

Fig. 6. The sweet potato latent virus-sweet potato (SPLV-sp) enzyme-linked immunosorbent assay (ELISA) kit was used to detect sweet potato and lotus samples. (A) SPLV-sp ELISA kit was used to detect sweet potato samples from field. (B) SPLV-sp ELISA kit was used to detect lotus samples from field. CP, coat protein; RT-PCR, reverse transcription polymerase chain reaction; SPLV-lotus, sweet potato latent virus from lotus. relative intensities of these points are shown by a bar graph (Fig. 4A). The relative value of the blank was set at 0 and then calculated the relative values of the other samples (Fig. 4A). The same samples were based on the ELISA results, and we plotted the bar graph (Fig. 4B), which was consistent with the results of the above dot blot, indicating that samples 1, 2, 3, 6, 9, 11, and 12 were positive samples. The $\mathrm{OD}_{405}$ value of the positive control was higher than that of the negative control, and the difference was significant. RT-PCR showed that detection results were consistent with dot blot analysis and ELISA (Fig. 4C), and all of which were effective for SPLV-lotus detection based on highthroughput, fast $\mathrm{CP}$ antiserum methods.

SPLV-lotus and sweet potato isolates appeared clearly distinction in serology. It has been previously reported in the literature that SPLV is serologically related to some potyviruses (Hammond et al., 1992). Therefore, we used SPLV-lotus CP antiserum to detect sweet potato samples. Among the results in eight sweet potato samples, we detected SPLV-sp positive sweet potato samples by RTPCR No. 1 to 6, while western blot detected only samples No. 2 and 5 (Fig. 5A). It was also found that the SPLVsp positive sweet potato samples obtained by RT-PCR were not detected by ELISA (Fig. 5B). There was a crossreaction between SPLV-lotus and SPLV-sp serum, but the reaction was not significant. At the same time, we also used the SPLV-sp ELISA kit (Qiaodu, Shanghai, China) to detect sweet potato and lotus samples. It was found that the SPLV-sp ELISA kit responded strongly to sweet potato samples (Fig. 6A), but the response to the lotus sample was relatively weak (Fig. 6B). Non-specific reaction tests were performed using soybean mosaic virus and turnip mosaic virus with SPLV-sp serum (Supplementary Fig. 1). These clear showed the serum can be used to specific detection of SPLV.

There are only few reports in the literature about the viral diseases in lotus, and the key pathogens that cause the reduction of lotus production have not been found. In this study, we developed high-throughput and sensitive detection methods namely ELISA and dot blot based on specific antibodies. This is the first study in which the serological technique has been used for the identification of SPLVlotus.

Traditionally, virus-specific antiserum is prepared by immunizing animals with isolated and purified virus particles as antigens, but it is difficult to rule out the effects of host proteins, which often makes the antiserum prepared with low titer and poor specificity. Using prokaryotic expression technology, we can obtain a large number of single 
proteins encoded by the virus, without low expression of non-structural proteins and poor stability. In this study, the SPLV-lotus CP gene was cloned and expressed by prokaryote, and the recombinant protein was digested and purified. New Zealand white rabbits were immunized to prepare specific polyclonal antibodies. The successful preparation of this antibody can lay the foundation for further research on SPLV-lotus serology detection kit.

Previously, we showed that the nucleotide similarity between SPLV-lotus and sweet potato isolates are around $76 \%$ (Wang et al., 2019), which is consistent with the classification criteria of the family Potyviridae (Wang et al., 2019; Wylie et al., 2017). Here, we found that SPLV-lotus has a weaker serological cross-reactivity with SPLV-sp, while SPLV-sp also has no strong response to lotus (Figs. 5 and 6). Thus, it is necessary to establish SPLV-lotus for the serological detection of lotus.

In summary, standard, sensitive and high-throughput assays such as western blot, dot blot and ELISA have been established through the preparation of SPLV-lotus CP antiserum. And we also found that SPLV-lotus antiserum (prepared in this study) cannot be used to detect SPLV isolated in sweet potato samples successfully, and SPLV-sweet potato antiserum (Qiaodu, Shanghai) has weak serological responses to lotus. Our research provides a variety of fast and reliable assays for SPLV-lotus isolates.

\section{Conflicts of Interest}

No potential conflict of interest relevant to this article was reported.

\section{Acknowledgments}

This work was supported by grants from the National Key Research and Development Program of China (2020YFD1000300), Key project at central government level: The ability establishment of sustainable use for valuable Chinese medicine resources (2060302), China Agriculture Research System (CARS-24) and National Natural Science Foundation of China (No. 31601604).

\section{Electronic Supplementary Material}

Supplementary materials are available at The Plant Pathology Journal website (http://www.ppjonline.org/).

\section{References}

Bhimji, A., Zaragoza, A. A., Live, L. S. and Kelley, S. O. 2013. Electrochemical enzyme-linked immunosorbent assay fea- turing proximal reagent generation: detection of human immunodeficiency virus antibodies in clinical samples. Anal. Chem. 85:6813-6819.

Boonham, N., Kreuze, J., Winter, S., van der Vlugt, R., Bergervoet, J., Tomlinson, J. and Mumford, R. 2014. Methods in virus diagnostics: from ELISA to next generation sequencing. Virus Res. 186:20-31.

Chiang, P. Y. and Luo, Y. Y. 2007. Effects of pressurized cooking on the relationship between the chemical compositions and texture changes of lotus root (Nelumbo nucifera Gaertn.). Food Chem. 105:480-484.

Clark, M. F. and Adams, A. N. 1977. Characteristics of the microplate method of enzyme-linked immunosorbent assay for the detection of plant viruses. J. Gen. Virol. 34:475-483.

Engvall, E. and Perlmann, P. 1971. Enzyme-linked immunosorbent assay (ELISA) quantitative assay of immunoglobulin G. Immunochemistry 8:871-874.

Hammond, J., Jordan, R. L., Larsen, R. C. and Moyer, J. W. 1992. Use of polyclonal antisera and monoclonal antibodies to examine serological relationships among three filamentous viruses of sweetpotato. Phytopathology 82:713-717.

He, Z., Chen, W., Chen, C., Liu, X. and Li, L. 2019. First report of Apple stem grooving virus infecting lotus (Nelumbo nucifera) in China. Plant Dis. 103:782.

Masuda, J.-I., Ozaki, Y. and Okubo, H. 2007. Rhizome transition to storage organ is under phytochrome control in lotus ( $\mathrm{Ne}$ lumbo nucifera). Planta 226:909-915.

Tsai, S. J., Hutchinson, L. J. and Zarkower, A. 1989. Comparison of dot immunobinding assay, enzyme-linked immunosorbent assay and immunodiffusion for serodiagnosis of paratuberculosis. Can. J. Vet. Res. 53:405-410.

Wang, H., Liu, X., Gan, H., Chen, W., Wu, P., Li, L. and He, Z. 2019. Genomic and biological characterization of a novel strain of Sweet potato latent virus isolated from lotus ( $\mathrm{Ne}$ lumbo nucifera Gaertn.). J. Plant Pathol. 101:1077-1084.

Wang, M., Abad, J., Fuentes, S. and Li, R. 2013. Complete genome sequence of the original Taiwanese isolate of Sweet potato latent virus and its relationship to other potyviruses infecting sweet potato. Arch Virol. 159:2189-2192.

Wylie, S. J., Adams, M., Chalam, C., Kreuze, J., López-Moya, J. J., Ohshima, K., Praveen, S., Rabenstein, F., Stenger, D., Wang, A., Zerbini, F. M. and ICTV Report Consortium. 2017. ICTV virus taxonomy profile: Potyviridae 98:352-354.

Xie, Y., Jiao, X., Zhou, X., Liu, H., Ni, Y. and Wu, J. 2013. Highly sensitive serological methods for detecting Tomato yellow leaf curl virus in tomato plants and whiteflies. Virol. J. $10: 142$.

Yu, H., Cheng, L., Yin, J., Yan, S., Liu, K., Zhang, F., Xu, B. and Li, L. 2013. Structure and physicochemical properties of starches in lotus (Nelumbo nucifera Gaertn.) rhizome. Food Sci. Nutr. 1:273-283.

Yu, X., Sheng, J. J., Zheng, X. W., Diao, Y., Zheng, X. F., Xie, K. Q., Zhou, M. Q. and Hu, Z. L. 2015. First report of Dasheen mosaic virus infecting lotus (Nelumbo nucifera) in China. Plant Dis. 99:1449. 